УДК 661.91

05.00.00 Технические науки

СОВЕРШЕНСТВОВАНИЕ ПРИЕМОВ
ОЧИСТКИ ГАЗА НА ОСНОВЕ ГАЗОВЫХ
СЕПАРАТОРОВ ВИХРЕВОГО ТИПА СГВ-7

Привалов Дмитрий Михайлович

Магистр

privaldo@mail.ru

Привалова Наталья Михайловна

к.х.н., доцент

dodoka57@mail.ru

Двадненко Марина Владимировна

к.х.н., доцент

meriru@rambler.ru

Кубанский Государственный Технологический

университет, Краснодар, Россия

Юрьев Эдуард Владимирович

Генеральный директор

ООО «НПО Вертекс», Краснодар, Россия

Настоящая статья посвящена обзору существующего сепарационного оборудования. Описан принцип работы и преимущества современной конструкции центробежновихревого сепаратора. Описана методика ремонта и модернизации устаревшего сепарационного оборудования

Ключевые слова: СЕПАРАЦИОННОЕ ОБОРУДОВАНИЕ, ЦЕНТРОБЕЖНОВИХРЕВОГО СЕПАРАТОР, СЕПАРАТОР ГРАВИТАЦИОННОГО ТИПА

Doi: 10.21515/1990-4665-130-019
UDC 661.91

Technical sciences

\section{PERFECTION OF METHODS OF GAS PURIFICATION ON THE BASIS OF SGV-7 GAS SEPARATORS OF VERTICAL TYPE}

Privalov Dmitry Mihailovich
Master
privaldo@mail.ru

Privalova Natalia Mikhailovna Cand.Chem.Sci., associate professor dodoka57@mail.ru

Dvadnenko Marina Vladimirovna Cand.Chem.Sci., associate professor meriru@rambler.ru

Kuban State University of Technology, Krasnodar, Russia

Yuryev Eduard Vladimirovich

General manager

NPO Vertex, Krasnodar, Russia

This article is devoted to the review of existing separation equipment. The principle of operation and advantages of a modern design of a centrifugal-vortex separator is described. The technique of repairing and modernization of obsolete separation equipment is described

Keywords: SEPARATING EQUIPMENT, CENTRIFUGAL-VORTEX SEPARATOR, SEPARATOR OF GRAVITATIONAL TYPE

Весь газ, добываемый на газовых месторождениях, транспортируемый по газотранспортной системе, обязательно проходит стадии очистки [1-3], в том числе на газовых сепараторах. Поэтому качество очистки газа и эффективность работы газовых сепараторов являются особенно актуальными для бесперебойного снабжения потребителей газа.

Меняющиеся условия эксплуатации и нестабильность параметров входного потока приводит к некачественной очистке газа на существующих газовых сепараторах. Это связано с тем, что их http://ej.kubagro.ru/2017/06/pdf/19.pdf 
конструкции рассчитаны на узкий диапазон эффективной работы и не способны динамично адаптироваться к реальным условиям работы. Это приводит к нестабильности показателей эффективности работы сепараторов и пылеуловителей и несоответствию требованиям к качеству очистки газа. Вследствие этого происходит накопление жидкости, которая остается в выходящем газовом потоке, в местных сопротивлениях, сужениях трубопровода, неровностях рельефа и прочих. Это приводит к потерям давления на преодоление газовым потоком этих препятствий. В целом вышеуказанные причины приводят к увеличению перепада давления на участке газопровода, что в свою очередь приводит к увеличению эксплуатационных и операционных затрат газотранспортного предприятия [4-6].

Основным сепарационным оборудованием, установленным сегодня на подавляющей части газодобывающих и газотранспортных объектов являются газовые сепараторы гравитационного типа рисунок 1. Это аппараты с известной низкой эффективностью работы.

Снижение эффективности работы гравитационных сепараторов происходит вследствие их конструктивных особенностей.

Так, диаметр сепаратора определяют по количеству газа, который пропускается через него и средней допустимой скорости газового потока по формуле:

$$
D_{s}=\sqrt{\frac{Q_{H} p_{0} z T_{c e n}}{67824 v_{c p} p_{1} T_{0}}},
$$

где, D - внутренний диаметр сепаратора м; Qн - пропускная способность сепаратора в $\mathrm{m}^{3} /$ сут; $\mathrm{p}_{0}$ - абсолютное атмосферное давление в н/ $\mathrm{M}^{2} ; \mathrm{z}$ - коэффициент сжимаемости; $\mathrm{T}_{\text {сеп }}-$ температура газа в сепараторе в К;. $\mathrm{v}_{\mathrm{cp}}$ - средняя оптимальная скорость движения газового в м/сек; $\mathrm{p}_{1}$ давление в сепараторе в н/ $\mathrm{M}^{2} ; \mathrm{T}_{0}-$ температура газа, $\mathrm{T}_{0}=293 \mathrm{~K}$. 
Диаметр гравитационного сепаратора рекомендуется определять по скорости осаждения капель жидкости расчетного диаметра.

$$
\omega=\left[\frac{4}{3}\left(\frac{\rho_{2} d}{\mu}\right)^{0.6} \frac{g d\left(\rho_{k}-\rho_{2}\right)}{18,5 \rho_{2}}\right]^{\frac{1}{1,4}},
$$

Расчеты показывают, что скорость осаждения капли диаметром 80 мкм в гравитационном сепараторе составляет - 0,08 м/с.

Условием работы вертикального сепаратора гравитационного типа, является условие, когда средняя скорость движения газового потока меньше скорости осаждения капли расчетного диаметра во встречном потоке $\mathrm{v}_{\mathrm{cp}}<\omega$.

Именно поэтому самые распространенные газовые сепараторы гравитационного типа имеют диаметры корпусов от 1000 мм.

Одним из эффективных способов решения проблемы качественной очистки газа от капельной жидкости и механических примесей является использование вихревых сепараторов.

Использование центробежно-вихревого эффекта в качестве основной сепарирующей силы позволяет не только «управлять» процессом сепарации, но и значительно сократить габариты сепарационного оборудования, поскольку основная сепарирующая сила будет выражаться по формуле:

$$
T=\frac{4 \pi \cdot R(\rho-\rho) v_{t}^{2}}{3 r}
$$

где $r$ - радиус траектории движения капли; $v_{t}$ - тангенциальная составляющая скорости движения капли; $R$ - радиус капли; $\rho_{l}, \rho$ плотность жидкости и среды.

Как видно из формулы 3, при уменьшении диаметра центробежного сепаратора, сепарирующая сила, действующая на каплю, растет. В жизни, это приводит к тому, что на сходные условия эксплуатации, сепараторы, 
использующие центробежно-вихревой эффект имеют значительно меньшие габариты и вес по сравнению с фильтр-сепараторами, сетчатыми или гравитационными,.

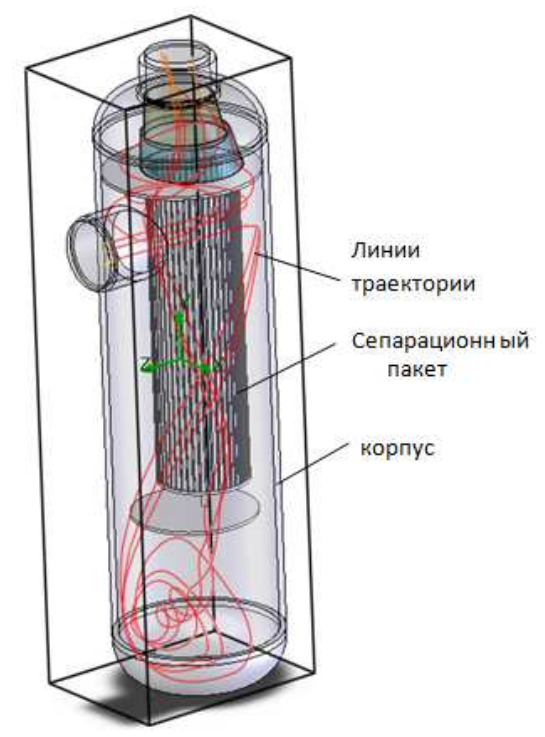

Рисунок 1. Расчетная модель газового сепаратора.

Эффективность сепарации газовых сепараторов гравитационного типа и сетчатых сепараторов также часто находится за пределами требуемых значений. Кроме этого, эффективность работы сепараторов с сетчатыми насадками по мере эксплуатации значительно ухудшается.

Подтверждением этому служат неоднократные замены сетчатых насадок гравитационных сепараторов, на встраиваемые сепараторы СГВ-7, проведенные ООО «НПО ВЕРТЕКС» по заявкам газотранспортных предприятий.

На рисунке 2 показана сетчатая насадка, отработавшая 2 года в конструкции гравитационного сетчатого сепаратора на ГРС. При этом следует учесть, что на ГРС поступает (должен поступать), как правило, предварительно очищенный газ, без содержания капельной жидкости и механических примесей. 


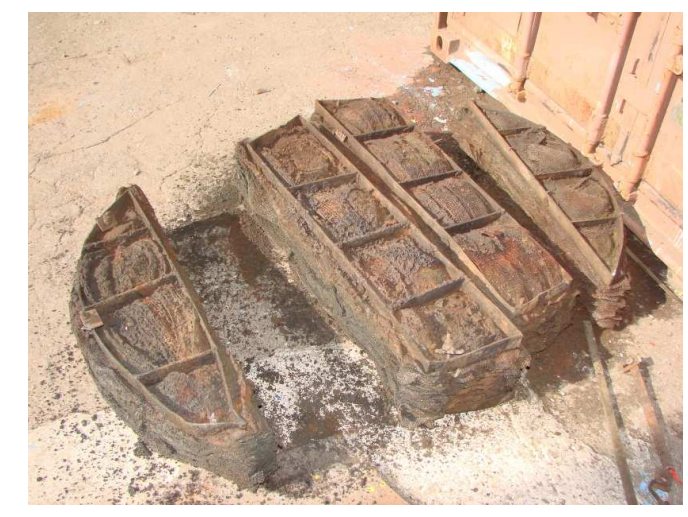

Рисунок 2. Коалесцирующий фильтрующий элемент сетчатого типа, извлеченный из сепаратора после 2-х лет работы.

На сегодняшний день предприятием ООО «НПО ВЕРТЕКС» разработаны, производятся и внедряются сепараторы СГВ-7 рисунок 3.

Сепаратор СГВ-7, предназначен для очистки газового потока и потока сжатого воздуха от механических примесей, капельной жидкости, находящейся во взвешенном состоянии и других, не растворенных примесей. Унос из аппарата составляет 4-6 мг/норм.м³.

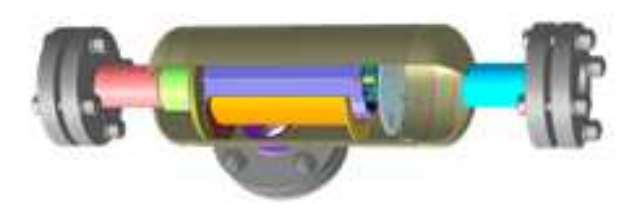

Рисунок 3. Сепаратор СГВ -7.

Газовый сепаратор СГВ-7 не имеет:

- фильтров, требующих частой замены;

- вращающихся и движущихся частей, нередко приводящих к отказам оборудования подобного типа;

- сложных внутренних конструктивных элементов, создающих трудности при обслуживании, ремонте и внутреннем осмотре аппарата, а также перепады давления при движении газожидкостного потока внутри сепаратора;

- других подобных недостатков, препятствующих эффективной работе и использованию сепаратора. 
Как известно, эффективность работы сепаратора можно измерять в относительных значениях (например в \%) или в абсолютном выражении (в $\left.\mathrm{M} / \mathrm{H} \cdot \mathrm{M}^{3}\right)$.

При измерении эффективности работы сепаратора в относительных значениях, она определяется по формуле:

$$
\eta=1-\frac{\omega_{1}}{\omega_{0}}
$$

где $\omega_{1}$ - объемное содержание жидкости в потоке на входе в сепаратор, равное:

$$
\omega_{1}=\int_{0}^{R m} V \cdot n_{0}(R) d R
$$

где $\omega_{0}$ - объемное содержание жидкости в потоке на входе в сепаратор, равное:

$$
\omega_{0}=\int_{0}^{\infty} V \cdot n_{0}(R) d R
$$

где $V=\frac{4}{3} \pi R^{3}$ - объем капли; $\mathrm{n} 0(\mathrm{R})$ - начальное распределение капель на входе; Rm - минимальны радиус тех капель, которые осядут в сепараторе.

Подставляя (5) и (6) в (4), получим

$$
\eta=1-\frac{4 \pi}{3 \omega_{0}} \int_{0}^{R m} R^{3} \cdot n_{0}(R) d R
$$

Однако при определении эффективности сепарации в относительных значениях часто допускается распространенная ошибка, поскольку такой коэффициент сильно зависит от количества жидкости на входе. При этом, если количество жидкости на входе будет мало, а количество жидкости на выходе у любого сепаратора величина постоянная, то и эффективность работы такого сепаратора будет не высокой, что само по себе абсурдно.

Поэтому более корректно определять эффективность работы сепаратора исходя из абсолютных значений количества жидкости на выходе из аппарата. 
Определить количество уноса жидкости из аппарата не сложно, достаточно использовать одну из нескольких методик определения уноса, например по методике определения прироста массы фильтрующего элемента.

Как было указано выше, сегодня процент внедрения нового оборудования на газотранспортных и газодобывающих предприятиях крайне низок. Это зачастую приводит к понижению качества очистки газа.

В свою очередь это делает проведение ремонта и модернизации существующего сепарационного оборудования особенно актуальной.

Сегодня существует методика ремонта и модернизации устаревшего сепарационного оборудования без нарушения целостности корпуса сосуда.

Суть методики заключается в том, что при ремонте сепараторов или пылеуловителей рисунок 4 не происходит нарушение целостности корпуса аппарата, а замена внутренних элементов происходит через существующие люк-лазы. При этом обвязка ремонтируемого сепаратора не меняется.
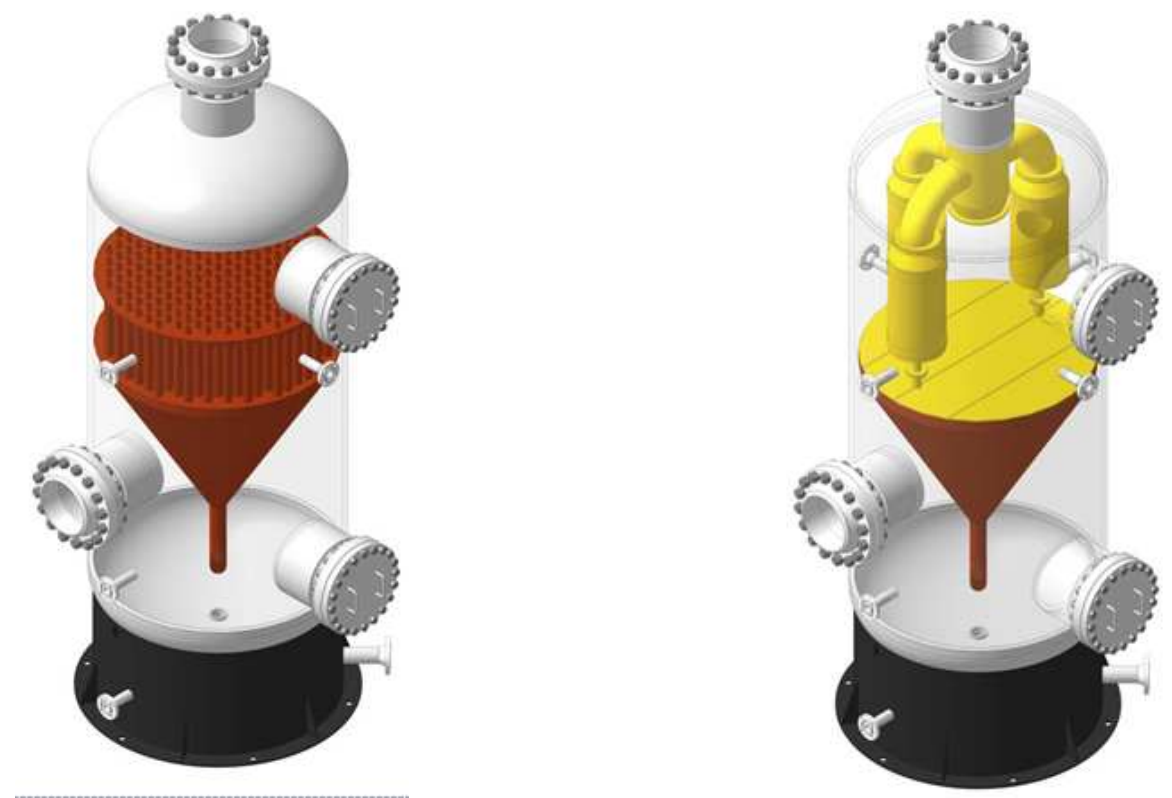

Пылеуловитель проекта ГП 105 Пылеуловитель проекта ГП 105 до проведения ремонта по после проведения ремонта по указанной методике указанной методике

Рисунок 4. Пылеуловитель ГП 105

Данный метод позволяет достичь максимальных результатов и имеет ряд преимуществ, а именно:

http://ej.kubagro.ru/2017/06/pdf/19.pdf 
- отсутствует необходимость изменения технологической обвязки трубопроводов аппарата,

- не требуется проект на выполнение указанных работ,

- работы по замене внутренних элементов проводятся на месте установки аппарата,

- отсутствует необходимость проведения дефектоскопии сварных соединений корпуса сосуда, так как монтаж производится к внутренним элементам аппарата не нагруженных давлением, не затрагивая стенок сосуда,

- сравнительно невысокая стоимость выполнения работ,

- короткие сроки выполнения работ.

Проведение ремонта и модернизации по указанной методике, с использованием сепарационных элементов СГВ-7 позволяет достичь максимальных результатов по эффективности очистки газа - унос жидкости из аппарата до 4 - 15 мг/н.м³, перепад давления на

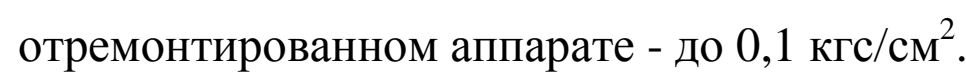

Продолжительность выполнения работ по ремонту и модернизации одного аппарата, без учета времени на оформление исполнительной документации, составляет до 14 - 18 рабочих дней с момента окончания подготовительных работ и подписания акта о выполнении владельцем аппарата мероприятий по безопасности труда.

Положительные отзывы газодобывающих и газотранспортных организаций и сравнительно невысокая стоимость проведения работ указывают на то, что данный способ восстановления работоспособности сепарационного оборудования является актуальным и целесообразным сегодня и останется таким в ближайшем будущем.

\section{Выводы}

1. Проанализирована сравнительная эффективность работы сепараторов гравитационного и центробежно-вихревого типов. 
2. Указаны недостатки существующих сепараторов гравитационного типа и фильтр-сепараторов.

3. Описан принцип работы и преимущества современной конструкции центробежно-вихревого сепаратора.

4. Описана методика ремонта и модернизации устаревшего сепарационного оборудования.

\section{Литература}

1. Привалова Н.М., Двадненко М.В., Шабанов А.Н. Каталитическая очистка газовых выбросов // Современные наукоемкие технологии. 2004. № 2. С. 56.

2. Некрасова А.А., Привалов Д.М., Двадненко М.В., Привалова Н.М Исследование сорбционных свойств фильтрующих материалов // Политематический сетевой электронный научный журнал Кубанского государственного аграрного университета. 2017. № 126. С. 266-275.

3. Боковикова Т.Н., Привалова Н.М., Двадненко М.В. Перспективы каталитической очистки газовых выбросов // Управление качеством: электронный журнал для местной промышленности. 2004. № 2.

4. Юрьев Э.В. Опыт проведения ремонта устаревшего сепарационного оборудования методом замены внутренних сепарационных элементов на газовый сепаратор СГВ-7 на объектах ООО «Газпром трансгаз - Кубань» // Материалы международной научно-практической конференции «Нафтегазоэнергетика: проблемы и перспективы». - м.Івано Франковск: 2009. с.55.

5. Привалов Д.М., Ксандопуло С.Ю., Привалова Н.М. Совершенствование приемов ресурсосбережения при утилизации попутного нефтяного газа. Тенденции развития науки и образования//Сборник научных трудов по результатам XIV международной научной конференции 31 мая 2016 г.Самара. Часть 1. Изд. НИЦ«ЛЖурнал», 2016.-52 с.

6. Привалов Д. М., Ксандопуло С. Ю., Привалова Н. М.. Проблемы эксплуатации существующей УКПГ «ВЯГП - ЕТЫ - Пуровское месторождение» ООО «Газпром Добыча Ноябрьск»//Тенденции развития науки и образования. Сборник научных трудов по результатам XIV международной научной конференции 31 мая 2016 г. Самара. Часть 1. Изд. НИЦ «Л-Журнал»., 2016. - 52 с.

\section{References}

1. Privalova N.M., Dvadnenko M.V., Shabanov A.N. Kataliticheskaja ochistka gazovyh vybrosov // Sovremennye naukoemkie tehnologii. 2004. № 2. S. 56.

2. Nekrasova A.A., Privalov D.M., Dvadnenko M.V., Privalova N.M Issledovanie sorbcionnyh svojstv fil'trujushhih materialov // Politematicheskij setevoj jelektronnyj nauchnyj zhurnal Kubanskogo gosudarstvennogo agrarnogo universiteta. 2017. № 126. S. 266-275. 
3. Bokovikova T.N., Privalova N.M., Dvadnenko M.V. Perspektivy kataliticheskoj ochistki gazovyh vybrosov // Upravlenie kachestvom: jelektronnyj zhurnal dlja mestnoj promyshlennosti. 2004. № 2.

4. Jur'ev Je.V. Opyt provedenija remonta ustarevshego separacionnogo oborudovanija metodom zameny vnutrennih separacionnyh jelementov na gazovyj separator SGV-7 na ob\#ektah OOO «Gazprom transgaz - Kuban'» // Materialy mezhdunarodnoj nauchnoprakticheskoj konferencii «Naftegazojenergetika: problemy i perspektivy». - m.Ivano Frankovsk: 2009. s.55.

5. Privalov D.M., Ksandopulo S.Ju., Privalova N.M. Sovershenstvovanie priemov resursosberezhenija pri utilizacii poputnogo neftjanogo gaza. Tendencii razvitija nauki i obrazovanija//Sbornik nauchnyh trudov po rezul'tatam XIV mezhdunarodnoj nauchnoj konferencii 31 maja 2016 g.Samara. Chast' 1. Izd. NIC«L-Zhurnal», 2016.-52 s.

6. Privalov D. M., Ksandopulo S. Ju., Privalova N. M.. Problemy jekspluatacii sushhestvujushhej UKPG «VJaGP - ETY - Purovskoe mestorozhdenie» OOO «Gazprom Dobycha Nojabr'sk»//Tendencii razvitija nauki i obrazovanija. Sbornik nauchnyh trudov po rezul'tatam XIV mezhdunarodnoj nauchnoj konferencii 31 maja 2016 g. Samara. Chast' 1. Izd. NIC «L-Zhurnal»., 2016. - 52 s. 